\title{
Humanized anti CCR4 antibody KW0761 targets HTLV-1 infected CD4+ CCR4+ and CD8+CCR4+ T cells to treat HAM/TSP
}

\author{
Yoshihisa Yamano ${ }^{1 *}$, Junji Yamauchi', Ariella Coler-Reilly', Tomoo Sato', Natsumi Araya', Naoko Yagishita', \\ Yasuo Kunitomo', Katsunori Takahashi ${ }^{1}$, Yuetsu Tanaka ${ }^{2}$, Hisanao Akiyama ${ }^{3}$, Yasuhiro Hasegawa ${ }^{3}$, \\ Atae Utsunomiya ${ }^{4}$
}

From 17th International Conference on Human Retroviruses: HTLV and Related Viruses

Trois Ilets, Martinique. 18-21 June 2015

Human T-lymphotropic virus type I (HTLV-1) can cause HTLV-1-associated myelopathy/tropical spastic paraparesis (HAM/TSP) and adult T cell leukemia/lymphoma (ATL). Since the prognosis for HAM/TSP patients is extremely poor, there is a strong demand for a novel therapeutic strategy, especially one which would effectively reduce HTLV-1 proviral load, which is well-correlated with disease prognosis. CD4+CCR4+ T cells are the main HTLV-1 reservoir, and the defucosylated humanized antiCCR4 antibody KW0761 has been approved in Japan as a treatment for ATL. KW0761 strongly binds to Fc $\gamma$ receptor IIIa (FcyRIIIa) on natural killer cells and elicits powerful antibody-dependent cellular cytotoxicity (ADCC) against the CCR4+ cells. In this study, we evaluated KW0761 as a treatment for HAM/TSP using primarily ex vivo cell cultures. In addition, given that KW0761 would also target CD8+CCR4+ T cells, we sought to determine how this would likely affect HAM/TSP patients by elucidating the role of CD8+CCR4+ T cells in HAM/TSP pathogenesis. When applied to cultures of PBMCs from HAM/ TSP patients ( $\mathrm{n}=11)$, KW0761 effectively reduced HTLV-1 proviral load $(56.4 \%$ mean reduction at $0.01 \mu \mathrm{g} / \mathrm{mL})$, spontaneous proliferation, and production of pro-inflammatory cytokines including IFN- $\gamma$. Like CD4+CCR4+ T cells, CD8+CCR4+ T cells from HAM/TSP patients exhibited high proviral loads and spontaneous IFN $-\gamma$ production, unlike their CCR4-counterparts. CD8+CCR4+ T cells from HAM/TSP patients contained more IFN- $\gamma+$ cells and less IL- $4+$ cells than those from healthy donors. Notably,
Tax-specific cytotoxic $\mathrm{T}$ lymphocytes that may help control the HTLV-1 infection were overwhelmingly CCR4-. In conclusion, we determined that $\mathrm{CD} 8+\mathrm{CCR} 4+\mathrm{T}$ cells as well as CD4+CCR4+ $\mathrm{T}$ cells are prime therapeutic targets for treating HAM/TSP, and that KW0761 shows promise as a new treatment. Based on the results of this study, we have begun conducting an investigator -led Phase I/IIa clinical trial to test the safety and efficacy of KW0761 on HAM/TSP patients (UMIN000012655).

\section{Authors' details}

'Department of Rare Diseases Research, Institute of Medical Science, St. Marianna University School of Medicine, Kawasaki, Kanagawa, Japan.

${ }^{2}$ Department of Immunology, Graduate School of Medicine, University of the Ryukyus, Okinawa, Japan. ${ }^{3}$ Division of Neurology, Department of Internal Medicine, St. Marianna University School of Medicine, Kawasaki, Kanagawa, Japan. ${ }^{4}$ Department of Hematology, Imamura Bun-in Hospital, Kagoshima, Japan.

Published: 28 August 2015

doi:10.1186/1742-4690-12-S1-O23

Cite this article as: Yamano et al: Humanized anti CCR4 antibody KW0761 targets HTLV-1 infected CD4+ CCR4+ and CD8+CCR4+ T cells to treat HAM/TSP. Retrovirology 2015 12(Suppl 1):O23.

\footnotetext{
* Correspondence: yyamano@marianna-u.ac.jp

'Department of Rare Diseases Research, Institute of Medical Science, St.

Marianna University School of Medicine, Kawasaki, Kanagawa, Japan

Full list of author information is available at the end of the article
} 